\title{
Impact of the introduction of thoracoscopic lobectomy for non-small cell lung cancer: a propensity score-matched analysis
}

\author{
Makoto Odaka, Yuki Noda, Yo Tsukamoto, Daiki Kato, Takamasa Shibasaki, Shohei Mori, Hisatoshi \\ Asano, Hideki Matsudaira, Makoto Yamashita, Toshiaki Morikawa \\ Department of Surgery, Jikei University School of Medicine, Tokyo, Japan \\ Contributions: (I) Conception and design: M Odaka; (II) Administrative support: M Odaka, T Morikawa; (III) Provision of study materials or patients: \\ M Odaka, Y Noda, Y Tsukamoto, D Kato, T Shibasaki, S Mori, H Asano, H Matsudaira, M Yamashita; (IV) Collection and assembly of data: M \\ Odaka, Y Noda, Y Tsukamoto, D Kato, T Shibasaki, S Mori, H Asano, H Matsudaira, M Yamashita; (V) Data analysis and interpretation: M Odaka; \\ (VI) Manuscript writing: All authors; (VII) Final approval of manuscript: All authors. \\ Correspondence to: Makoto Odaka, MD. Department of Surgery, Jikei University School of Medicine, 3-19-18 Nishishinbashi, Minatoku, Tokyo 105- \\ 0003, Japan. Email: mak@jikei.ac.jp.
}

\begin{abstract}
Background: The present study evaluated the impact of the introduction of thoracoscopic lung lobectomy (TL) for non-small cell lung cancer at our institution.

Methods: This study retrospectively compared surgical and oncological outcomes in the period before and after the introduction of TL for non-small cell lung cancer. Propensity score-matched analysis was performed with respect to baseline patient variables and tumor characteristics.

Results: Patients were divided into two groups: those who underwent lung lobectomy in the period before (BI group, $\mathrm{n}=261$ ) and after (AI group, $\mathrm{n}=261$ ) the introduction of TL. The proportion of TLs at our institution increased from $1.3 \%$ in the BI group to $93 \%$ in the AI group. The AI group experienced a longer duration of surgery, lesser intraoperative blood loss, and a significantly shorter postoperative hospital stay (POHS). There were no significant differences in postoperative complications between the two groups. The median follow-up period was 50 months in both groups. No significant differences were observed between the BI and AI groups with respect to 5 -year overall survival (OS) $(76.1 \%$ and $71.7 \%$, respectively; $\mathrm{P}=0.1973)$ and disease-free survival (DFS) (67.6\% and 66.1\%, respectively; $\mathrm{P}=0.4071)$. On multivariate analysis, pathological N1-2 status was an independent predictor of survival. AI group and TL showed no independent association with survival.

Conclusions: The introduction of TL represented a positive change at our institution owing to decreased invasiveness and oncological equivalence of the surgical treatment for non-small cell lung cancer.
\end{abstract}

Keywords: Thoracoscopy; lung cancer; disease-free survival (DFS)

Submitted Jun 30, 2018. Accepted for publication Jul 20, 2018.

doi: $10.21037 /$ jtd.2018.07.107

View this article at: http://dx.doi.org/10.21037/jtd.2018.07.107

\section{Introduction}

Thoracoscopic surgery is widely used for the treatment of non-small cell lung cancer (1-3). At our institution, we experienced a major change with the introduction of thoracoscopic lung lobectomy (TL) in 2005. Several studies have compared surgical outcomes of thoracoscopic surgery with those of open surgery. However, the impact of the introduction of TL for non-small cell lung cancer has not been investigated at an institutional level. In the present study, we evaluated the impact of the introduction of TL for non-small cell lung cancer at our institution. Propensity score-matched analysis was used for a comprehensive comparison of surgical and oncological outcomes before and after the introduction of TL.

\section{Methods}

We retrospectively reviewed the records of 916 consecutive 
patients with non-small cell lung cancer (clinical stage IA-IIIA) who underwent anatomical lung lobectomy between 1996 and 2016 at the Jikei University Hospital, Japan. Thoracoscopic surgery was introduced at our institution in July 2005. Prior to this, open lobectomy via standard thoracotomy was the main surgical modality for the treatment of non-small cell lung cancer. After the introduction of thoracoscopic surgery, it has been used as the main approach in a vast majority of patients. For the comparative study, patients were divided into two groups: those who underwent lung lobectomy before the introduction of TL for non-small cell lung cancer (BI group, $\mathrm{n}=622$ ) and those who underwent lung lobectomy after the introduction of TL (AI group, $n=294$ ). Patients who underwent TL in the last 2 years were excluded because of the short follow-up period. From 2005 to 2016, 1,335 TLs were performed at our institution by two boardcertified dedicated thoracic surgeons and eight trainee surgeons under the supervision of expert surgeons. Patient characteristics, surgical procedures, and surgical and oncological outcomes were compared between the BI and AI groups.

The indications for TL were as follows: clinical T (cT) $1-3$, cN $0-1$, clinical stage IA-IIIA, single N2 disease, and Eastern Cooperative Oncology Group performance status of $0-1$. Conventional open lobectomy (OL) was performed in patients who did not meet the TL criteria or in those with suspected clinical hilar lymph node involvement that could hamper the thoracoscopic handling. Few patients with cT4 disease underwent OL. Multiple N2 diseases were excluded from the criteria. Because this was an intention-totreat analysis, patients in whom thoracoscopic surgery was converted to open surgery were included in the TL group. TL with curative intent was approved by our local ethics committee, and written informed consent was obtained from all patients.

We classified the $\mathrm{cN}$ status as positive when the shortest diameter was $\geq 10 \mathrm{~mm}$ on computed tomography (CT). Positron emission tomography was used for the clinical evaluation of most patients with $\mathrm{N} 2$ disease. The 7 th edition of the tumor-node-metastasis classification (4) was used for tumor staging. The final diagnosis, status of surgical margins, and TNM stage were confirmed by a histopathological examination of resected specimens for all patients. None of the patients experienced any major postoperative complication, and all patients were in a stable general condition at the time of discharge. Postoperatively, patients were reevaluated every 6 months with a chest CT for 5 years and once annually thereafter.

\section{Surgical procedures}

Patients undergoing TL were placed in the lateral position and a double-lumen endotracheal tube for split-lung ventilation was used for anesthesia. Patients underwent TL completely under the thoracoscope. The affected side of the thoracic cavity was approached via a 4- or 5-port technique. A thoracoscope was introduced at an oblique angle of $30^{\circ}$ through the port in the 4th intercostal space at the posterior axillary line and fixed with an exclusive arm. The working ports were placed in the $3 \mathrm{rd}$, 4th, and 6th lateral intercostal spaces at the anterior axillary line. If required, an additional working port was placed in the 6th intercostal space at the posterior axillary line. The major vascular branches and pulmonary parenchyma were transected using a stapler. The minor vascular branches and small bronchi were ligated with an energy device and/or suture. The specimen was removed in a plastic retrieval bag.

OL was performed using the conventional procedure via a posterolateral incision. Systematic hilar and mediastinal lymph node dissection was usually performed during both TL and OL.

\section{Statistical analysis}

Data are reported as median (range). JMP 12 (SAS Institute Inc., Cary, NC, USA) was used for statistical analyses. Between-group differences with respect to categorical variables were assessed using the Pearson $\chi^{2}$ test and those with respect to continuous variables were assessed using the Wilcoxon test. Propensity scores were calculated using a logistic regression model that included the following variables: age, sex, clinical $\mathrm{T}$ and $\mathrm{N}$ status, tumor size, histology, and postoperative adjuvant therapy. Scores were generated from the model for caliper matching using a caliper distance of 0.05 without replacement and 1:1 matching with control. The KaplanMeier method was used to plot survival curves, and the log-rank test was used to assess between-group differences with respect to overall survival (OS) and disease-free survival (DFS). Univariate and multivariate analyses using a Cox proportional hazard analysis were also performed to identify correlates of OS and DFS in both groups. Survival time was defined as the time from surgery until death, the last follow-up, or recurrence. $\mathrm{P}<0.05$ was considered statistically significant. 
Table 1 Characteristics, surgical approach and clinical tumor status in patients who underwent lung lobectomy for non-small cell lung cancer in the period before and after the introduction of thoracoscopic lobectomy

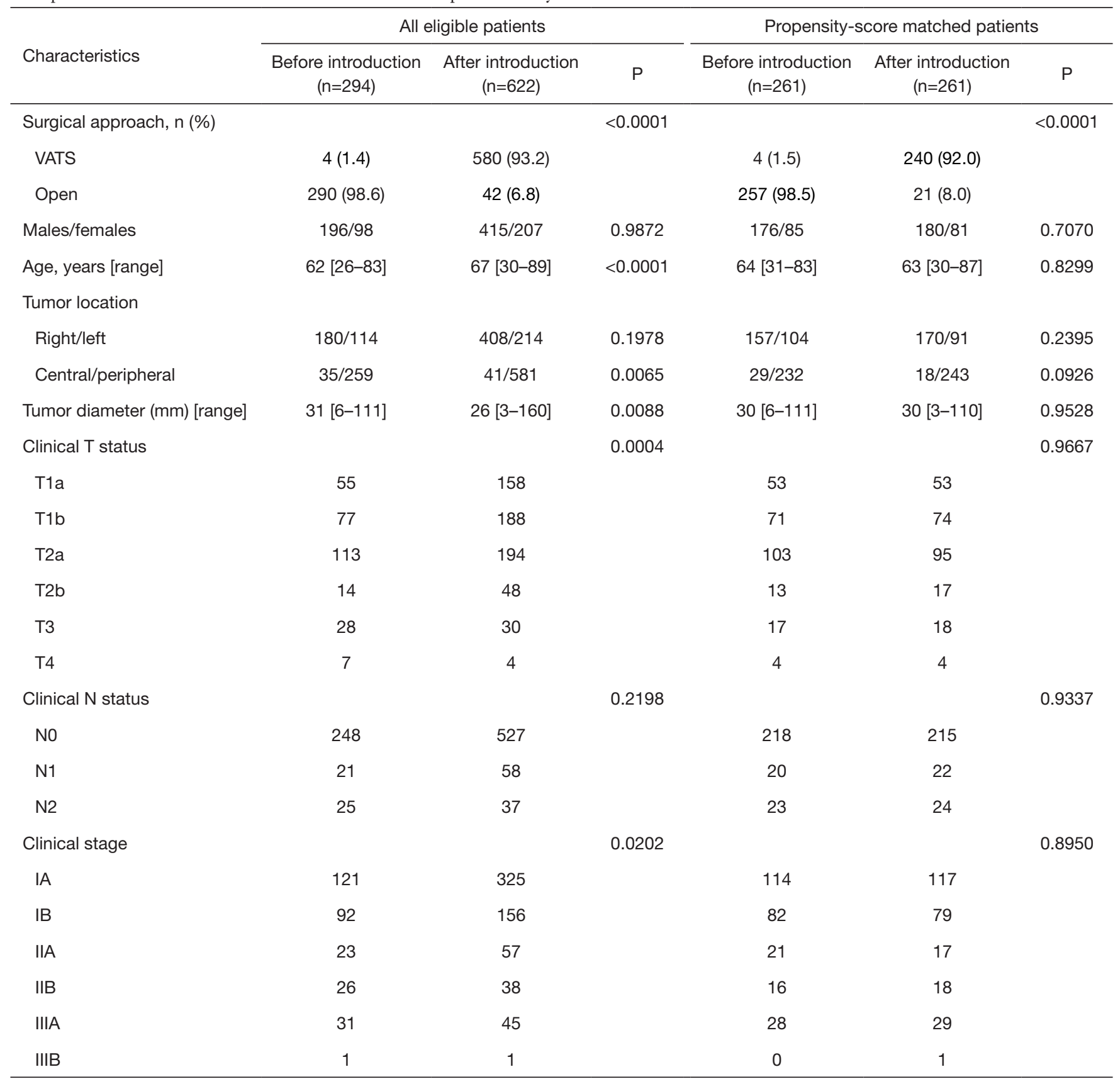

POHS, postoperative hospital stay.

\section{Results}

\section{Patient characteristics and surgical outcomes}

Patient characteristics, surgical procedures and clinical tumor status are presented in Table 1. Of the 1,335 patients who underwent surgical treatment during the study reference period, 916 (68.6\%; BI group, $\mathrm{n}=294$ and $\mathrm{AI}$ group, $\mathrm{n}=622$ ) qualified under the study selection criteria. Significant differences were observed between the two groups with respect to patient characteristics, tumor location, $\mathrm{cT}, \mathrm{N}$ status, and clinical stage. The proportion of TLs at our institution increased from $1.3 \%$ in the BI 
group to almost $93 \%$ in the AI group. In the AI group, 580 patients underwent TL and 42 underwent OL. Fortytwo patients who underwent conventional OL either did not qualify the criteria for TL or were suspected of having clinical hilar lymph node involvement that could hamper the thoracoscopic handling. Four patients had T4 tumor, three required lobectomies with chest wall resection, and four had large tumors. Nine patients with cT1/2 had hilar enlarged lymph nodes. Nineteen patients underwent OL because of the preference of the surgeon. Because this was an intention-to-treat analysis, 41 patients in whom TL was converted to OL were considered to have undergone TL. Most reasons for conversion were associated with the handling of the pulmonary artery.

After propensity score matching of patients with complete variables, there were 261 patients each in the $\mathrm{BI}$ and AI groups. There were no significant differences in patient characteristics, tumor location, tumor size, $\mathrm{cT}$, $\mathrm{N}$ status, or clinical stage between the two groups. Of the 261 patients in the AI group, 240 (92.0\%) underwent the thoracoscopic approach without mini-thoracotomy. Of these 240 patients, $218(90.8 \%)$ underwent a completed thoracoscopic procedure without conversion to open surgery. Conversions to open surgery occurred in 22 (9.2\%) patients, with the reasons for conversion being bleeding from the pulmonary artery in four patients, difficult dissection of the pulmonary artery in seven, severe adhesion, or fissure less of lobes. Perioperative surgical outcomes and pathological tumor status are presented in Table 2. The AI group had a longer duration of surgery $(\mathrm{P}<0.0001)$, lower intraoperative blood loss $(\mathrm{P}=0.0017)$, and significantly shorter postoperative hospital stay (POHS; $\mathrm{P}<0.0001)$. There were no significant differences in postoperative complications between the two groups $(\mathrm{P}=0.0752)$. The most common postoperative complication in the AI group was air leakage lasting for $\geq 7$ days $(n=16)$. The BI group showed a higher incidence of respiratory complications, atelectasis, and pneumonia than the AI group. There was no significant difference in patients who received postoperative adjuvant therapy between the two groups. No significant between-group differences were observed with respect to tumor histology, pathologic $\mathrm{N}$ status and $\mathrm{p}$ stage.

\section{Oncological outcomes}

The median overall follow-up period was 50 months (BI group, 50 months; AI group, 50 months). There was no significant difference between the BI and AI groups with respect to the 5-year OS $(76.1 \%$ and $71.7 \%$, respectively; $\mathrm{P}=0.1973$; Figure $1 A)$ and DFS $(67.6 \%$ and $66.1 \%$, respectively; $\mathrm{P}=0.4071 ;$ Figure $1 \mathrm{~B})$. A significant difference was observed in the 5 -year OS between patients who underwent TL and those who underwent OL via standard thoracotomy ( $78 \%$ and $70.1 \%$, respectively; $\mathrm{P}=0.0248$; Figure $2 A$ ). However, no significant difference was observed between the two approaches with respect to DFS (68.3\% and $65.5 \%$, respectively; $\mathrm{P}=0.1509$; Figure $2 B$ ). A total of 82 patients in the $\mathrm{BI}$ group and 78 in the $\mathrm{AI}$ group experienced recurrence $(\mathrm{P}=0.7041)$.

\section{Prognostic factors}

We sought to identify variables that showed a significant influence on OS and DFS. On univariate analysis, male sex, central tumor, tumor size of $\geq 30 \mathrm{~mm}, \mathrm{cT} 2 \mathrm{ab}-4$, pN1-2, SCC, and TL showed a significant influence on OS, whereas male sex, central tumor, tumor size of $\geq 30 \mathrm{~mm}$, cT2ab-4, and pN1-2 showed a significant influence on DFS (Table 3). These variables were included in the Cox proportional hazard analysis. Pathological N1-2 status was found to be an independent predictor of OS and DFS (Table 4), whereas AI group and TL were not independently associated with OS and DFS.

\section{Discussion}

Over the last two decades, thoracoscopic surgery has been introduced in several institutions. TL is expected to be less invasive than OL for non-small cell lung cancer (1,3,5-9). Several studies have demonstrated the safety and feasibility of TL (1,5-9). Furthermore, the advantage of TL in terms of oncological outcomes has also been reported (5,6,10-14). From an institutional perspective, it is important to assess the impact of the introduction of TL for non-small cell lung cancer. The primary purpose of the present study was to evaluate the impact of the introduction of TL for non-small cell lung cancer at our institution. We compared surgical and oncological outcomes before and after the introduction of TL for non-small cell lung cancer. Most surgical results in the AI group (with the exception of surgical duration) were better than those in the BI group. The present study showed that AI was associated with a significantly shorter POHS. Patients in the BI group experienced more respiratory complications than those in the AI group. Major postoperative complications were similar between the 
Table 2 Perioperative surgical outcomes and pathological tumor status in patients who underwent lung lobectomy for non-small cell lung cancer in the period before and after the introduction of thoracoscopic lobectomy

\begin{tabular}{|c|c|c|c|c|c|c|}
\hline Characteristics & \multicolumn{3}{|c|}{ All eligible patients } & \multicolumn{3}{|c|}{ Propensity-score matching patients } \\
\hline Blood loss (mL) [range] & $162[5-1704]$ & $100[0-3100]$ & $<0.0001$ & $160[5-1704]$ & $100[0-2500]$ & 0.0017 \\
\hline Complications, n (\%) & $61(20.7)$ & 105 (16.9) & 0.156 & $58(22.2)$ & $42(16.1)$ & 0.0752 \\
\hline Pneumonia & 18 & 12 & & 12 & 6 & \\
\hline Atelectasis & 10 & 1 & & 13 & 0 & \\
\hline Chylothorax & 0 & 7 & & 0 & 3 & \\
\hline Bronchial fistula & 1 & 4 & & 0 & 2 & \\
\hline Pyothorax & 1 & 3 & & 1 & 1 & \\
\hline Arrhythmia & 3 & 11 & & 3 & 3 & \\
\hline Mortality, n (\%) & $0(0.0)$ & $4(0.6)$ & & $0(0.0)$ & $2(0.8)$ & - \\
\hline Adjuvant therapy, n (\%) & $109(37.1)$ & $133(21.4)$ & 0.0005 & $92(35.2)$ & $94(36.0)$ & 0.855 \\
\hline Histology & & & 0.2472 & & & 0.9046 \\
\hline $\mathrm{N} 1$ & 43 & 71 & & 39 & 39 & \\
\hline $\mathrm{N} 2$ & 42 & 62 & & 37 & 44 & \\
\hline Pathological stage & & & $<0.0001$ & & & 0.5895 \\
\hline IA & 87 & 276 & & 84 & 95 & \\
\hline IB & 78 & 150 & & 69 & 58 & \\
\hline$\| A$ & 39 & 79 & & 36 & 38 & \\
\hline IIB & 29 & 48 & & 18 & 22 & \\
\hline IIIA & 53 & 66 & & 47 & 45 & \\
\hline IIIB & 8 & 3 & & 7 & 3 & \\
\hline
\end{tabular}

POHS, postoperative hospital stay; complications, postoperative complications; conversion, conversion to open thoracotomy. 

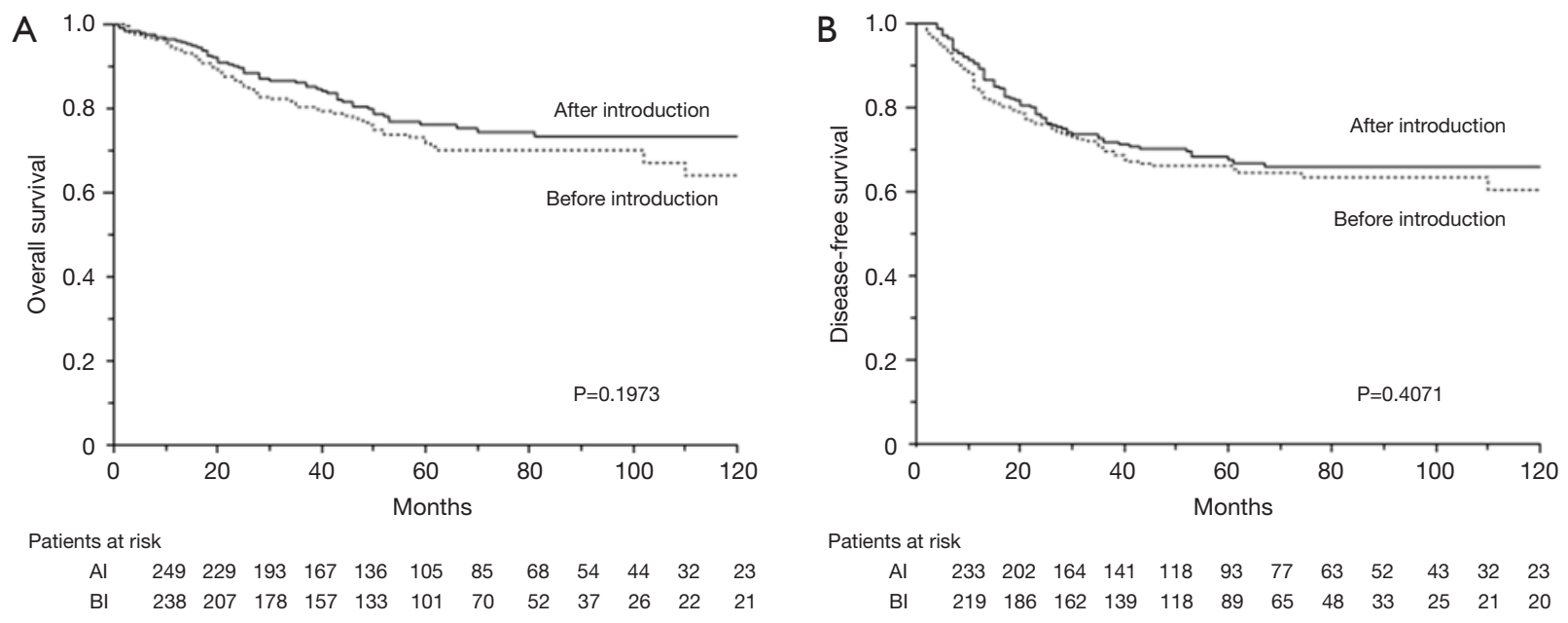

Figure 1 Overall survival (A) and disease-free survival (B) curves of propensity score-matched patients in the period before and after the introduction of thoracoscopic lobectomy groups.
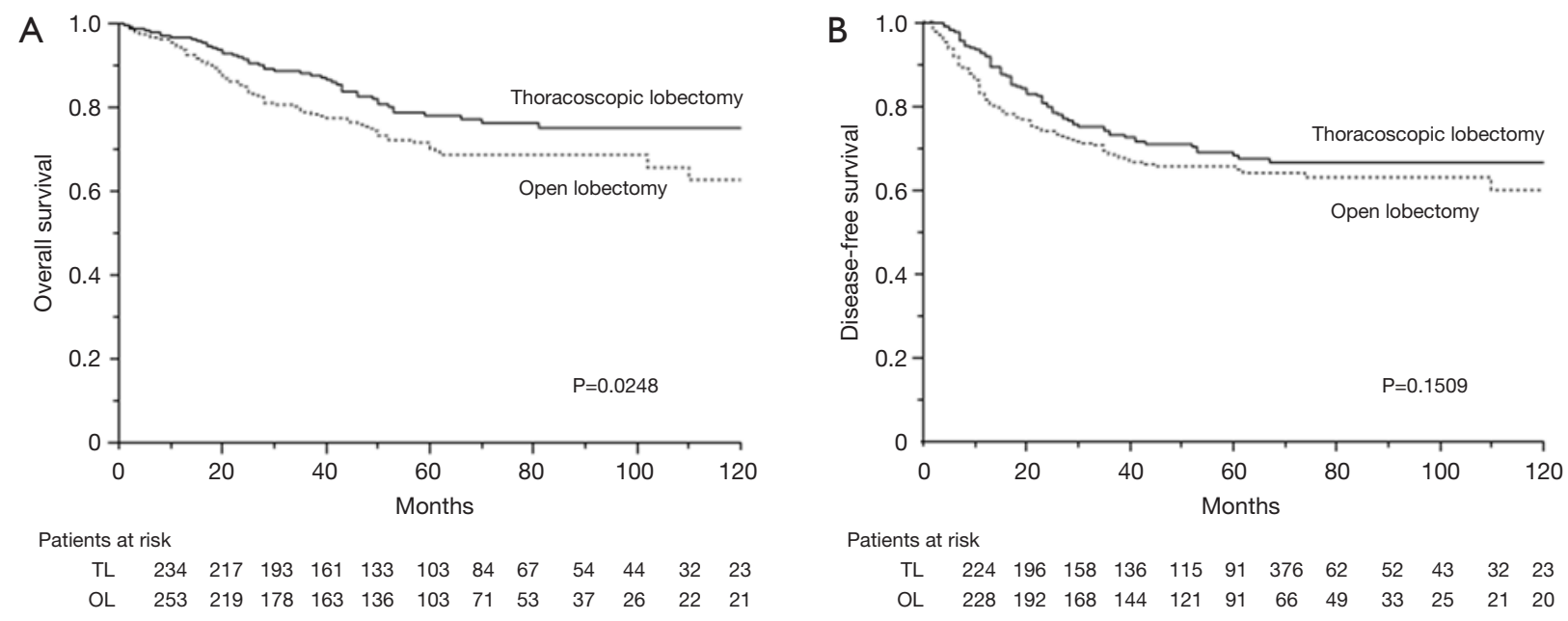

Figure 2 Overall survival (A) and disease-free survival (B) curves of propensity score-matched patients in the thoracoscopic and open lobectomy groups.

two groups, and prolonged air leak was the most common complication. The postoperative complication rate $(16 \%)$ and mortality $(0.7 \%)$ were comparable with those reported from other studies. Previous studies on thoracoscopic surgery for lung cancer have reported that TL favorably compares with thoracotomy, with complication rates ranging from $15.3 \%$ to $32 \%$ and perioperative mortality ranging from $0.2 \%$ to $3.6 \%(1,7,15-20)$. The current literature shows that TL is a considerably less invasive surgical treatment for non-small cell lung cancer. The introduction of TL helped improve surgical outcomes at our institution.

Our conversion rate $(9.1 \%)$ was comparable with that reported in the literature. Published conversion rates from video-assisted thoracic surgery to thoracotomy range from $1.6 \%$ to $17 \%(1,7,15-20)$. At our institution, most conversions were attributable to difficulties in handling the pulmonary artery. Therefore, conversion should be dictated by safety concerns during the procedure. Patients who required conversion did not have larger tumors than those who did not require one. Thus, it seems difficult to preoperatively identify patients who are likely to require 
Table 3 Results of univariate analysis showing factors associated with survival of patients who underwent lung lobectomy for non-small cell lung cancer

\begin{tabular}{|c|c|c|c|c|c|c|}
\hline Characteristics & \multicolumn{3}{|c|}{ 5-year OS } & \multicolumn{3}{|c|}{ 5-year DFS } \\
\hline Male & 1.8881 & $1.24-2.97$ & 0.0025 & 1.6071 & $1.13-2.33$ & 0.0074 \\
\hline Left & 0.1176 & $0.77-1.60$ & 0.5511 & 1.0211 & $0.73-1.39$ & 0.8973 \\
\hline Central & 2.6936 & $0.59-4.29$ & 0.0004 & 1.7997 & $1.09-2.80$ & 0.0224 \\
\hline cT2ab-4 & 2.6487 & $1.80-3.95$ & $<0.0001$ & 2.1677 & $1.57-3.01$ & $<0.0001$ \\
\hline pN1-2 & 4.9538 & $3.43-7.18$ & $<0.0001$ & 4.3218 & $3.15-5.92$ & $<0.0001$ \\
\hline SCC & 1.6689 & $1.11-2.45$ & 0.0143 & 1.3500 & $0.93-1.91$ & 0.1090 \\
\hline Thoracoscopic lobectomy & 0.6595 & $0.45-0.94$ & 0.0245 & 0.7978 & $0.58-1.08$ & 0.1524 \\
\hline
\end{tabular}

OS, overall survival; DFS, disease-free survival; $\mathrm{Cl}$, confidence interval; HR, hazard ratio; SCC, squamous cell carcinoma.

Table 4 Results of multivariate analysis showing factors associated with survival of patients who underwent lung lobectomy for non-small cell lung cancer

\begin{tabular}{|c|c|c|c|c|c|c|}
\hline Characteristics & \multicolumn{3}{|c|}{ 5-year OS } & \multicolumn{3}{|c|}{ 5-year DFS } \\
\hline Male & 1.1587 & $0.97-2.43$ & 0.0643 & 1.4170 & $0.99-2.06$ & 0.0554 \\
\hline Central & 1.2514 & $0.71-2.08$ & 0.4144 & 0.9703 & $0.57-1.54$ & 0.9036 \\
\hline Size $\geq 30 \mathrm{~mm}$ & 0.7907 & $0.36-2.05$ & 0.5998 & 1.4838 & $0.67-3.98$ & 0.3495 \\
\hline pN1-2 & 4.1077 & $2.80-6.05$ & $<0.0001$ & 3.8867 & $2.80-5.39$ & $<0.0001$ \\
\hline SCC & 1.1511 & $0.74-1.75$ & 0.5233 & - & - & - \\
\hline Thoracoscopic lobectomy & 0.7521 & $0.51-1.08$ & 0.1329 & - & - & - \\
\hline
\end{tabular}

OS, overall survival; DFS, disease-free survival; $\mathrm{Cl}$, confidence interval; HR, hazard ratio; SCC, squamous cell carcinoma.

open thoracotomy without an intraoperative thoracoscopic assessment. In a study by Hennon et al. (21), the conversion rate for advanced-stage lung cancers was higher than that for early-stage lung cancers. Reported disadvantages of TL include difficulty manipulating relatively large tumors and lymph node dissection $(5,22)$. Technical difficulties of TL for advanced lung cancer result from less mobilization of tumor and constrained working space. Furthermore, the need for multiple thoracoscopic instruments in the operative field poses technical difficulties. Our thoracoscopic technique ensures a satisfactory view for the surgeon despite deep and narrow spaces.

The next purpose of this study was to assess oncological outcomes around the period of introduction of TL for non-small cell lung cancer. Long-term survival rates represent the best indicators of oncological success. Survival in the AI group was largely comparable to that in the BI group. However, OS of patients who underwent TL was significantly longer than that of patients who underwent OL, which suggested that the introduction of TL contributed to the improvement of oncological outcomes. Other studies that have assessed differences in survival rates between TL and OL have yielded variable results $(23,24)$. Several authors $(5,6,10-14)$ have reported a statistically significant survival advantage of TL. Recent studies have reported comparable oncological outcomes 
of TL and OL (5,6,10-14). Taioli et al. (25) indicated that patients undergoing TL had a $5 \%$ survival advantage over $\mathrm{OL}$ at 5 years. Among the other advantages, this survival advantage is also likely attributable to the minimallyinvasive nature of surgery that limits cytokine induction and helps preserve the immune system, thereby improving longterm OS $(5,6,13,26)$. Recent therapeutic advances including postoperative chemotherapy or molecular-targeted therapy may have contributed to the trend toward improved OS with TL. Our results indicated that OS and DFS were equivalent after TL or OL.

In our Cox hazard multivariate analysis, OS and DFS were significantly correlated with pathological lymph node status. The pathological $\mathrm{N}$ status was revealed as an independent prognostic factor for OS and DFS. In a study by Lee $e t$ al. (10), advanced pathological N1-2 disease was found to be an independent predictor of worse DFS, which is consistent with our results. Stephens et al. (11) reported TL as an independent predictor of survival. In our study, $\mathrm{TL}$ was not found to be a significant prognostic factor on multivariate analysis.

The retrospective study design and single-center scope are key limitations of our study that may have introduced an element of selection bias. Surgical procedures may improve over time regardless of the surgical approach. Moreover, because there was a considerable change in the ratio of $\mathrm{TL}$ and OL before and after the introduction of TL at our institution, the learning curve of surgeons at our institution is certainly a form of selection bias. In addition, advances in chemotherapy over time represent another element of selection bias. However, propensity score matching analysis based on patient characteristics helped minimize the selection bias. In the absence of prospective randomized studies, the selection bias for thoracoscopic surgery cannot be easily eliminated. Therefore, larger prospective randomized clinical studies are required to obtain more definitive evidence of the efficacy of TL.

\section{Conclusions}

The introduction of TL at our institution represented a positive change owing to decreased invasiveness and oncological equivalence of the surgical treatment for nonsmall cell lung cancer.

\section{Acknowledgements}

None.

\section{Footnote}

Conflicts of Interest: The authors have no conflicts of interest to declare.

Ethical Statement: The study was approved by our local ethics committee (IRB number: 18-232 4894), and written informed consent was obtained from all patients.

\section{References}

1. McKenna RJ, Houck W, Fuller CB, et al. Video-assisted thoracic surgery lobectomy: experience with 1,100 cases. Ann Thorac Surg 2006;81:421-5; discussion 425-6.

2. Hartwig MG, D'Amico TA. Thoracoscopic lobectomy: the gold standard for early-stage lung cancer. Ann Thorac Surg 2010;89:S2098-101.

3. Scott WJ, Allen MS, Darling G, et al. Video-assisted thoracic surgery versus open lobectomy for lung cancer: a secondary analysis of data from the American College of Surgeons Oncology Group Z0030 randomized clinical trial. J Thorac Cardiovasc Surg 2010;139:976-81.

4. The Japanese Lung Cancer Society. General rule for clinical and pathological records of lung cancer. Tokyo: Kanehara \& Co. Ltd, 2010:87-94.

5. Whilston BA, Groth SS, Duval SJ, et al. Surgery for earlystage non-small cell lung cancer: a systematic review of the video-assisted thoracoscopic surgery versus thoracotomy approaches to lobectomy. Ann Thorac Surg 2008;86:200816; discussion 2016-8.

6. Yan TD, Black D, Bannon PG, et al. Systematic review and meta-analysis of randomized and nonrandomized trials on safety and efficacy of video-assisted thoracic surgery lobectomy for early-stage non-small cell lung cancer. J Clin Oncol 2009;27:2553-62.

7. Onaitis MW, Petersen RP, Balderson SS, et al. Thoracoscopic lobectomy is a safe and versatile procedure: experience with 500 consecutive patients. Ann Surg. 2006;244:420-5.

8. Shigemura N, Akashi A, Nakagiri T, et al. Complete versus assisted thoracoscopic approach: A prospective randomized trial comparing a variety of video-assisted thoracoscopic lobectomy techniques. Surg Endosc 2004;18:1492-7.

9. Swanson SJ, Herndon JE 2nd, D'Amico TA, et al. Videoassisted thoracic surgery lobectomy: report of CALGB 39802-a prospective, multi-institution feasibility study. J Clin Oncol 2007;25:4993-7.

10. Lee PC, Nasar A, Port JL, et al. Long-term survival after 
lobectomy for non-small cell lung cancer by video-assisted thoracic surgery versus thoracotomy. Ann Thorac Surg 2013:96:951-60.

11. Stephens N, Rice D, Correa A, et al. Thoracoscopic lobectomy is associated with improved short-term and equivalent oncological outcomes compared with open lobectomy for clinical Stage I non-small-cell lung cancer: a propensity-matched analysis of 963 cases. Eur J Cardiothorac Surg 2014;46:607-13.

12. Cao C, Zhu ZH, Yan TD, et al. Video-assisted thoracic surgery versus open thoracotomy for non-small-cell lung cancer: a propensity score analysis based on a multi-institutional registry. Eur J Cardiothorac Surg 2013:44:849-54.

13. Kaseda S, Aoki T, Hangai N, et al. Better pulmonary function and prognosis with video-assisted thoracic surgery than with thoracotomy. Ann Thorac Surg 2000;70:1644-6.

14. Hanna WC, de Valence M, Atenafu EG, et al. Is videoassisted lobectomy for non-small-cell lung cancer oncologically equivalent to open lobectomy? Eur J Cardiothorac Surg 2013;43:1121-5.

15. Flores RM, Park BJ, Dycoco J, et al. Lobectomy by videoassisted thoracic surgery (VATS) versus thoracotomy for lung cancer. J Thorac Cardiovasc Surg 2009;138:11-8.

16. Villamizar NR, Darrabie MD, Burfeind WR, et al. Thoracoscopic lobectomy is associated with lower morbidity compared with thoracotomy. J Thorac Cardiovasc Surg 2009;138:419-25.

17. Daniels LJ, Balderson SS, Onaitis MW, et al. Thoracoscopic lobectomy: a safe and effective strategy for patients with stage I lung cancer. Ann Thorac Surg 2002;74:860-4.

18. Villamizar NR, Darrabie M, Hanna J, et al. Impact of $\mathrm{T}$ status and $\mathrm{N}$ status on perioperative outcomes after

Cite this article as: Odaka M, Noda Y, Tsukamoto Y, Kato D, Shibasaki T, Mori S, Asano H, Matsudaira H, Yamashita M, Morikawa T. Impact of the introduction of thoracoscopic lobectomy for non-small cell lung cancer: a propensity scorematched analysis. J Thorac Dis 2018;10(8):4985-4993. doi: 10.21037/jtd.2018.07.107 thoracoscopic lobectomy for lung cancer. J Thorac Cardiovasc Surg 2013;145:514-20.

19. Gonzalez D, de la Torre M, Paradela M, et al. Videoassisted thoracic surgery lobectomy: 3-year initial experience with 200 cases. Eur J Cardiothorac Surg 2011;40:e21-8.

20. Marty-Ané CH, Canaud L, Solovei L, et al. Videoassisted thoracoscopic lobectomy: an unavoidable trend? A retrospective single-institution series of 410 cases. Interact Cardiovasc Thorac Surg 2013;17:36-43.

21. Hennon M, Sahai RK, Yendamuri S, et al. Safety of thoracoscopic lobectomy in locally advanced lung cancer. Ann Surg Oncol 2011;18:3732-6.

22. Denlinger CE, Fernandez F, Meyers BF, et al. Lymph node evaluation in video-assisted thoracoscopic lobectomy versus lobectomy by thoracotomy. Ann Thorac Surg 2010;89:1730-5; discussion 1736.

23. Farjah F, Wood DE, Mulligan MS, et al. Safety and efficacy of video-assisted versus conventional lung resection for lung cancer. J Thorac Cardiovasc Surg 2009; 137:1415-21.

24. Yang X, Wang S, Qu J. Video-assisted thoracic surgery (VATS) compares favorably with thoracotomy for the treatment of lung cancer: a five-year outcome comparison. World J Surg 2009;33:1857-61.

25. Taioli E, Lee DS, Lesser M, et al. Long-term survival in video-assisted thoracoscopic lobectomy vs open lobectomy in lung-cancer patients: a meta-analysis. Eur J Cardiothorac Surg 2013;44:591-7.

26. Ng CS, Wan S, Hui CW, et al. Video-assisted thoracic surgery lobectomy for lung cancer is associated with less immuno chemokine disturbances than thoracotomy. Eur J Cardiothorac Surg 2007;31:83-7. 